\title{
Asymmetry parameter and total cross section for the photodetachment of the metastable $\mathrm{Be}^{-} 1 s^{2} 2 s 2 p^{24} P$ state
}

\author{
Christos Sinanis, Yannis Komninos, and Cleanthes A. Nicolaides \\ Theoretical and Physical Chemistry Institute, National Hellenic Research Foundation, 48 Vas. Constantinou Avenue, \\ 11635 Athens, Greece \\ (Received 27 December 1994)
}

\begin{abstract}
We report the results of calculations of the asymmetry parameter and of the cross section for the photodetachment of the metastable state $\mathrm{Be}^{-} 1 s^{2} 2 s 2 p^{2}{ }^{4} P$, for photoelectron energies above the $\mathrm{Be} 1 s^{2} 2 s 2 p^{3} P^{o}$ threshold of $0.74-2.74 \mathrm{eV}$. The calculations were based on configuration-interaction-type wave functions, where the final scattering state is obtained via the solution to all orders of multichannel reaction $(K)$ matrices. Very good agreement is observed with the recent measurement of Pegg et al. [Phys. Rev. A 50, 3861 (1994)]
\end{abstract}

PACS number(s): $32.80 . \mathrm{Fb}$

In a recent publication, Pegg et al. [1] reported the results of measurements of the single-photon detachment cross section $\sigma$ and of the asymmetry parameter $\beta$ at photon energy $\hbar \omega=2.076 \mathrm{eV}$ for the process

$$
\begin{array}{r}
\mathrm{Be}^{-} 1 s^{2} 2 s 2 p^{2}{ }^{4} P+\hbar \omega \rightarrow \mathrm{Be} 1 s^{2} 2 s 2 p^{3} P^{o}+e^{-} \\
(\varepsilon s \text { and } \varepsilon d),
\end{array}
$$

where $\varepsilon=1.815 \mathrm{eV}$, according to the experimental value for the detachment threshold [2]. The $\sigma$ was measured to be $31 \pm 3 \mathrm{Mb}$, in serious disagreement with the only previously reported measurement, by Bae and Peterson [3], who found $\sigma=9 \pm 5 \mathrm{Mb}$. The $\beta$ was measured to be $0.49 \pm 0.02$. No other measurements or theoretical results exist for these properties.

The observed discrepancy between the two measurements and the lack of any theoretical predictions on the cross section and angular distribution of process (1) have led us to undertaking the calculation of $\sigma(\varepsilon)$ and $\beta(\varepsilon)$ in the photon energy range $1.0-3.0 \mathrm{eV}$. This range includes the one previously examined [3] (1.65-2.34), and corresponds to photoelectron energies above the $\mathrm{Be} 1 s^{2} 2 s 2 p^{3} P^{o}$ threshold of $0.74-2.74 \mathrm{eV}$.

The $\mathrm{Be}^{-} 1 \mathrm{~s}^{2} 2 s 2 p^{24} P$ state belongs to the class of excited states of atomic negative ions, which are bound. (For a recent review see Ref. [4].) As with many other such states, in the Hartree-Fock (HF) approximation this state is unbound. However, the fact that symmetry does not allow any hole-filling pair correlations (which would cause decay), and that the correlation energy of the three $\mathrm{Be}^{-} L$-shell electrons $\left(2 s 2 p^{2}{ }^{4} P\right)$ is larger than that of the $\mathrm{Be}\left(2 s 2 p^{3} P^{o}\right)$ pair, turns the $\mathrm{Be}^{-} 1 s^{2} 2 s 2 p^{24} P$ state into a discrete one [5,6]. Its first observation and identification were reported in [3,7]. Its existence was also verified from the observation [8] of the $\mathrm{Be}^{-} 1 s^{2} 2 p^{3}{ }^{4} S^{o}-1 s^{2} 2 s 2 p^{2}{ }^{4} P$ transition, whose possibility, wavelength, and probability had been predicted theoretically [6].

The present calculations were carried out in the framework of the state-specific theory of photoionization processes [9], which uses separately optimized wave functions that are expansions over symmetry-adapted bound and scattering configurations, whose coefficients are obtained by configuration-interaction (CI) methods with appropriate boundary conditions. The two quantities $\sigma(\varepsilon)$ and $\beta(\varepsilon)$ were computed from the definitions of Dill and Fano [10] (for reviews on the definition and calculation of photoionization observables see Refs. [11]-[13]):

$$
\begin{gathered}
\sigma(\varepsilon)=\sum_{j_{t}} \sigma\left(j_{t}\right), \\
\beta(\varepsilon)=\frac{1}{\sigma(\varepsilon)} \sum_{j_{t}} \sigma\left(j_{t}\right) \beta\left(j_{t}\right),
\end{gathered}
$$

where $j_{t}$ represents the possible magnitudes of the angularmomentum transfer. In the present problem (process 1), $j_{t}=1,2$. For $j_{t}=1$, representing the parity-favored case,

$$
\begin{gathered}
\beta(1)=\frac{\left|S_{d}(1)\right|^{2}-2 \sqrt{2} \operatorname{Re}\left[S_{d}(1) S_{s}(1)^{*}\right]}{\left|S_{d}(1)\right|^{2}+\left|S_{s}(1)\right|^{2}}, \\
\sigma(1)=\pi\left(\left|S_{d}(1)\right|^{2}+\left|S_{s}(1)\right|^{2}\right),
\end{gathered}
$$

while for $j_{t}=2$, the parity-unfavored case,

$$
\begin{gathered}
\beta(2)=-1, \\
\sigma(2)=5 / 3 \pi\left[\left|S_{d}(2)\right|^{2}\right] .
\end{gathered}
$$

The $S_{l}\left(j_{t}\right)$ are the scattering amplitudes defined by

$$
\begin{aligned}
S_{l_{c}}\left(j_{t}\right)= & (4 \pi / c)^{1 / 2} \sum_{L=1,2}(2 L+1)^{1 / 2}\left\{\begin{array}{ccc}
1 & l_{c} & L \\
1 & 1 & j_{t}
\end{array}\right\} \\
& \times\left\langle\Psi_{i}\left\|d^{(1)}\right\| \Psi_{l_{c}, L}^{-}\right\rangle,
\end{aligned}
$$

where $d^{(1)}$ is the dipole operator (length or velocity form), $l_{c}$ is the angular momentum of the free orbital, and $\Psi_{l_{c}, L}^{-}(\varepsilon)$ are the incoming wave functions for the three open channels $\varepsilon s^{4} P^{o}, \varepsilon d^{4} P^{o}, \varepsilon d^{4} D^{o}$.

Specifically, $\sigma(\varepsilon)$ and $\beta(\varepsilon)$ were calculated by implementing the following computational steps: 
(i) Initial state. The wave function of the initial state, $\Psi_{i}$, has the form

$$
\Psi_{i}=\Phi_{i}^{0}+X_{i}
$$

where $\Phi_{i}^{0}$ is a numerical multiconfigurational HF (MCHF) [14] function consisting of all configurations ( $1 s^{2}$ is frozen) that can be constructed from the Fermi-sea set [4] and references therein) of $n=2$ and $n=3$ orbitals, and $X_{i}$ is the variationally obtained remaining $L$-shell correlation correction, representing single, double, and triple excitations from the HF function that are orthogonal to $\Phi_{i}^{0}$. The final $\Psi_{i}(44$ configurations) was obtained from a nonorthonormal $\mathrm{CI}$ (NONCI) [4] with a total energy of $E_{i}=-14.5277$ a.u., which is the same as that obtained by Weiss [5].

(ii) Final state. The final-state wave function may have ${ }^{4} P^{o}$ or ${ }^{4} D^{o}$ total symmetry. The latter is obtained only if the free electron is a $d$ wave. The former is obtained from both $s$ and $d$ waves coupled to the ${ }^{3} P^{o}$ core. In this case, the physics involves three open channels, two of which interact. The free orbitals $\varepsilon s^{4} P^{o}, \varepsilon d^{4} P^{o}$, and $\varepsilon d^{4} D^{o}$ are obtained in the frozen-core HF approximation numerically [15], and are coupled to the core state $\mathrm{Be} 1 s^{2} 2 s 2 p^{3} P^{o}$, whose wave function was obtained at the MCHF level with six configurations $(2 s 2 p, 2 p 3 d, 2 p 3 s, 3 p 3 d, 3 s 3 p$, and $3 d 4 f)$. Electron correlation does not affect this state much, either in the wave function or in the energy. The coefficient of $(2 s 2 p)$ is 0.994 , the HF energy is -14.5115 a.u., while the MCHF energy is -14.51840 a.u.

Before the final CI in the continuum (CIC), closed channels were included as well to allow mainly for polarization. Thus, the following Be states were included, all represented by their own MCHF functions: $1 s^{2} 2 s 3 s^{3} s, 1 s^{2} 2 s 3 d^{3} D$, $1 s^{2} 2 p^{2}{ }^{3} P$. The MCHF energy differences between these states and the main one, $1 s^{2} 2 s 2 p^{3} P^{o}$, are in good agreement with the experimental ones. ${ }^{3} S-{ }^{3} P^{o}: \Delta E_{\text {th }}$ $=0.136$ a.u., $\Delta E_{\text {expt }}=0.137$ a.u.; ${ }^{3} D-{ }^{3} P^{o}: \Delta E_{\mathrm{th}}=0.183$ a.u., $\Delta E_{\text {expt }}=0.183$ a.u.; ${ }^{3} P-{ }^{3} P^{o}: \Delta E_{\text {th }}=0.173$ a.u., $\Delta E_{\text {expt }}=0.172$ a.u. The calculations for the $(2 s 2 p)^{3} P^{o} \varepsilon d^{4} D^{o}$ open channel were done in the presence of the $(2 s 3 d)^{3} D \varepsilon p,(2 s 3 d)^{3} D \varepsilon f,\left(2 p^{2}\right)^{3} P \varepsilon p$, and $\left(2 p^{2}\right)^{3} P \varepsilon f$ closed channels, while for the $(2 s 2 p)^{3} P^{o} \varepsilon s{ }^{4} P^{o}$ and $(2 s 2 p){ }^{3} P^{o} \varepsilon d^{4} P^{o}$ open channels the $(2 s 3 s)^{3} S \varepsilon p,(2 s 3 d)^{3} D \varepsilon p,(2 s 3 d)^{3} D \varepsilon f$, and $\left(2 p^{2}\right)^{3} P \varepsilon p$ closed channels were included.

Our approach to the computation of the multichannel final-state scattering wave functions has been presented before [9]. It leads to a CIC method that aims at the calculation of the relevant reaction $(K)$ matrices to all orders using statespecific correlated wave functions. The method allows the systematic incorporation of open and closed channels, of valence states, and of Rydberg levels.

The important element in the CIC method is the calculation of the $K$ matrix, in terms of which the $\Psi^{-}$of Eq. (8) is obtained. This is given by

$$
\begin{aligned}
K_{i j}(\varepsilon ; E)= & W_{i j}(\varepsilon ; E)+\sum_{l} P \int d \varepsilon^{\prime} W_{i l}\left(\varepsilon, \varepsilon^{\prime}\right) \frac{1}{E-\varepsilon^{\prime}} \\
& \times K_{l j}\left(\varepsilon^{\prime} ; E\right),
\end{aligned}
$$

where $W_{i j}\left(\varepsilon, \varepsilon^{\prime}\right)$ is the residual interaction between zerothorder channels $u_{i}(\varepsilon), u_{j}\left(\varepsilon^{\prime}\right)$,

$\left\langle u_{i}(\varepsilon)|H-E| u_{j}\left(\varepsilon^{\prime}\right)\right\rangle=W_{i j}\left(\varepsilon ; \varepsilon^{\prime}\right)+(\varepsilon-E) \delta_{i j} \delta\left(\varepsilon-\varepsilon^{\prime}\right)$.

The solution of Eq. (10) is achieved by transforming it into a set of linear algebraic equations. Thus, the nonsingular part of the integrant in the principal-value integral is fitted by a low-degree polynomial and the integration is performed analytically as in the Filon quadrature. Both the on- and the off-energy shell $K$ matrices are used in the calculation of the dipole matrix elements:

$$
D^{-} \equiv\left\langle\Psi_{0}\left|d^{(1)}\right| \Psi^{-}\right\rangle=D[1-i \pi K]^{-1},
$$

where

$D_{j}(E)=d_{j}(E)+\sum_{i} P \int d \varepsilon d_{i}(\varepsilon) \frac{1}{E-\varepsilon} K_{i j}(\varepsilon, E)$,

where $d_{j}(E)$ is the zeroth-order dipole matrix elements $\left\langle\Psi_{0}\left|d^{(1)}\right| u_{j}(E)\right\rangle$. Accurate interpolation to energies other than those of the chosen energy mesh is performed by the so-called Nystrom interpolation scheme [16]. Accordingly,
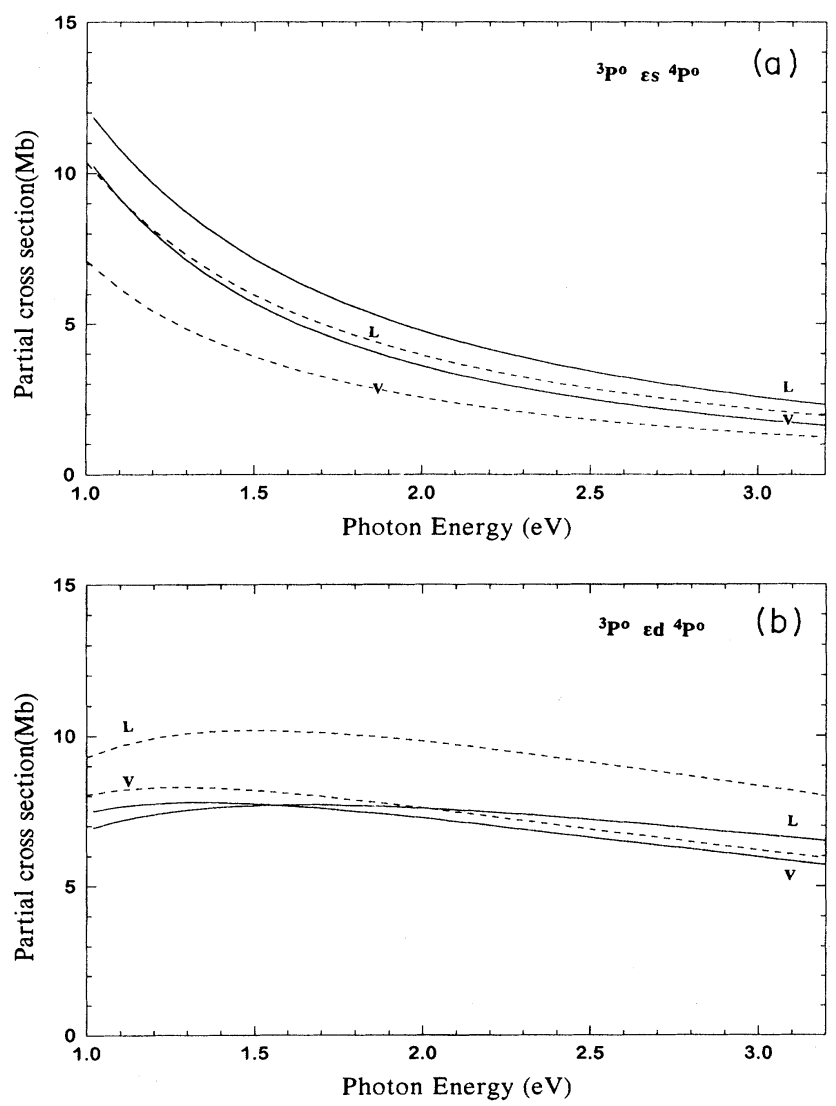

FIG. 1. (a) Partial photodetachment cross section for the $\varepsilon s{ }^{4} P^{o}$ channel (this work). HF results (----), with interchannel coupling (-). (b) Partial photodetachment cross section for the $\varepsilon d^{4} P^{o}$ channel. HF results (---), with interchannel coupling (-). 

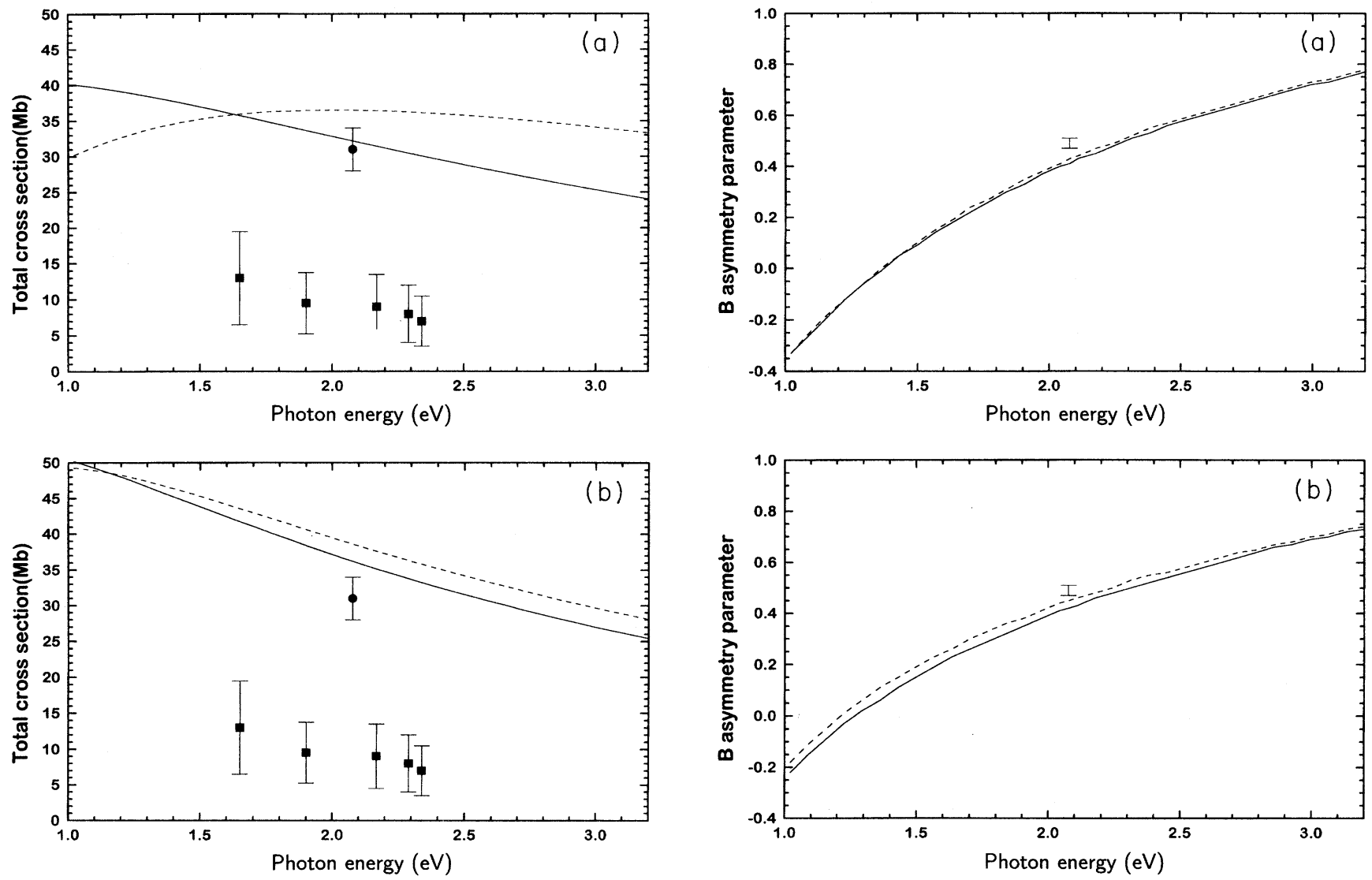

FIG. 2. (a) Total photodetachment cross section for process (1) in the HF approximation (this work). Length (----); velocity (-). Experiment: circles, Pegg et al. [1]; squares, Bae and Peterson [3]. (b) Total photodetachment cross section for process (1) from the state-specific theory (see text). Length (----); velocity (-). Experiment: circles, Pegg et al. [1]; squares, Bae and Peterson [3].

one interpolates the zeroth-order quantities, e.g., $d_{j}$ and $W_{i j}$, while the correlated quantities $D_{j}$ and $K_{i j}$ are obtained from the integral equations (10) and (13).

The following sets of scattering orbitals were used. For the open channels, 150 orbitals were used for each channel, from threshold to 4.0 a.u. For the energies $0.0-0.5$ a.u., we computed 100 orbitals, i.e., the number of energy points in this region was higher. For reasons of computational economy, the closed channels were divided into two categories. The first contained the states with a nonvanishing dipole matrix element with the initial state, i.e., $(2 s 3 s){ }^{3} S$ $\varepsilon p^{4} P^{o}, \quad\left(2 p^{2}\right)^{3} P \varepsilon p^{4} P^{o}, \quad(2 s 3 d)^{3} D \varepsilon p^{4} P^{o}, \quad\left(2 p^{2}\right)^{3} P$ $\varepsilon p{ }^{4} D^{o}$, and $(2 s 3 d)^{3} D \varepsilon p^{4} D^{o}$. For these, orbitals for 120 energies were calculated. The orbitals of the second category, $(2 s 3 d)^{3} D \quad \varepsilon f^{4} P^{o}, \quad\left(2 p^{2}\right)^{3} P \quad \varepsilon f^{4} D^{o}, \quad$ and $(2 s 3 d)^{3} D^{o}$ $\varepsilon f^{4} D^{o}$, were calculated for 75 energies, up to 4.0 a.u. In computing the interaction matrix elements as well as the dipole transition matrix elements, the nonorthonormality between the separately optimized basis functions for all the states involved was computed explicitly.

We report two sets of results. The first was obtained from calculations that used only HF functions for initial and final states and only open channels without interchannel coupling.

FIG. 3. (a) Asymmetry parameter for process (1) in the HF approximation (this work). Length (----); velocity (-). Experiment: Pegg et al. [1]. (b) Asymmetry parameter for process (1) from the state-specific theory (see text). Length (----); velocity (-). Experiment: Pegg et al. [1].

Experimental energy differences were used, since in the HF approximation the initial state is unbound. The second set represents our complete calculations, with electron correlation in the initial and final states, with polarization, and with interchannel coupling. (The $\varepsilon d^{4} D^{o}$ channel is about four times more important than the $\varepsilon s, \varepsilon d^{4} P^{o}$ channels.) All energies are obtained in an $a b$ initio manner. The results are shown in Figs. 1(a)-3(b).

Figures 1(a) and 1(b) present the results on the partial cross sections for the ${ }^{4} P^{o}$ channel, which has interchannel coupling. The dashed lines show the HF results in the length $(L)$ and velocity $(V)$ forms, and the full lines show the results after the inclusion of interchannel coupling. In both cases the initial state was represented by the correlated wave function. Figures 2(a) and 2(b) present the results for the total cross section. In Fig. 2(a), the calculation was done in the HF approximation. In Fig. 2(b) both initial and final results are correlated as discussed above (final results). The dashed lines correspond to the length form. It is indeed impressive that for this system the velocity form gives very reasonable results even in the HF approximation, where the $\varepsilon d^{4} D^{o}$ channel dominates. Finally, Figs. 3(a) and 3(b) present the results for the asymmetry parameter, without [Fig. 3(a)] and with [Fig. 3(b)] electron correlation in the 
initial and final states. Again it is seen that the effect of electron correlation and interchannel coupling for the (weak) ${ }^{4} P^{o}$ channel is small.

In conclusion, the state-specific theory of multichannel photodetachment, which was implemented here, has allowed the quantitative prediction of partial and total cross sections for process (1). This process is essentially dominated by the $\varepsilon d^{4} D^{o}$ channel, whose contribution is described well in the HF approximation. As regards the recently revealed discrepancy between the results of two different experimental methods $[1,3]$, the present theory shows a clear preference for the measurements of Pegg et al. [1] [Figs. 2(b) and 3(b)].
[1] D. J. Pegg, C. Y. Tang, J. R. Wood, J. Dellwo, and G. D. Alton, Phys. Rev. A 50, 3861 (1994).

[2] C. Y. Tang, J. R. Wood, D. J. Pegg, J. Dellwo, and G. D. Alton, Phys. Rev. A 48, 1983 (1993).

[3] Y. K. Bae and J. R. Peterson, Phys. Rev. A 30, 2145 (1984).

[4] C. A. Nicolaides, G. Aspromallis, and D. R. Beck, J. Mol. Struct. 199, 283 (1989).

[5] A. W. Weiss, Phys. Rev. 166, 70 (1968).

[6] D. R. Beck, C. A. Nicolaides, and G. Aspromallis, Phys. Rev. A 24, 3252 (1981); D. R. Beck and C. A. Nicolaides, Int. J. Quantum Chem. S18, 467 (1984).

[7] K. Bethge, E. Heinecke, and H. Baumann, Phys. Lett. 23, 542 (1966); H. J. Kaiser, E. Heinecke, H. Baumann, and K. Bethge, Z. Phys. 243, 46 (1971).

[8] J. O. Gaardsted and T. Andersen, J. Phys. B 22, L51 (1989).
[9] Y. Komninos and C. A. Nicolaides, Phys. Rev. A 34, 1995 (1986); Z. Phys. 4, 301 (1987).

[10] D. Dill and U. Fano, Phys. Rev. Lett. 29, 1203 (1972); U. Fano and D. Dill, Phys. Rev. A 6, 185 (1972).

[11] A. F. Starace, in Corpuscles and Radiation in Matter, edited by W. Melhorn, Encyclopedia of Physics Vol. 31 (SpringerVerlag, Berlin, 1982), p. 1.

[12] S. T. Manson and A. F. Starace, Rev. Mod. Phys. 54, 389 (1982).

[13] V. Schmidt, Rep. Prog. Phys. 55, 1483 (1992).

[14] C. Froese-Fischer, Comput. Phys. Commun. 14, 145 (1978).

[15] G. N. Bates, Comput. Phys. Commun. 8, 220 (1974).

[16] B. Noble, in The State of the Art in Numerical Analysis, edited by D. A. Jacobs (Academic Press, New York, 1977). 